\title{
Evaluating the Association of Heme and Heme Metabolites with Lung Cancer Bioenergetics and Progression
}

\section{Hooda J, Alam MM and Zhang L*}

Department of Molecular and Cell Biology, Center for Systems Biology, University of Texas at Dallas, Mail Stop RL11, 800 W, Campbell Road, Richardson, TX 75080, USA

\begin{abstract}
Emerging experimental data increasingly show that despite the enhanced glycolytic flux, many types of cancer cells exhibit intensified oxygen consumption or mitochondrial respiration. Even under hypoxia, cancer cells can maintain oxidative phosphorylation at a substantial rate. Heme is a central factor in oxygen utilization and oxidative phosphorylation. It serves as a prosthetic group in many proteins and enzymes involved in mitochondrial respiration. Notably, our recent work showed that non-small-cell lung cancer (NSCLC) cells and xenograft tumors exhibit substantially increased levels of an array of proteins promoting heme synthesis, uptake and function. These proteins include the rate-limiting heme biosynthetic enzyme ALAS, transporter proteins, and various types of oxygen-utilizing hemoproteins such as cytoglobin and cytochromes. In contrast, lowering heme biosynthesis and uptake, like lowering mitochondrial respiration, effectively reduced oxygen consumption, cancer cell proliferation, migration and colony formation. Therefore, elevated heme function and flux are likely key features of NSCLC cells and tumors. Based on this observation, we decided to further ascertain the relationship between heme and lung cancer. We extracted heme and its metabolites from various NSCLC cancer cells and measured the levels of heme in these cells. We also measured the rates of oxygen consumption in various cancer cells and compared them to the levels of heme in these cells. We expect that these experimental results will enable us to determine the extent to which heme and heme metabolites impact cancer cell bioenergetics and progression.
\end{abstract}

Keywords: Heme; Metabolites; Lung cancer; Glucose; Glutamine; NSCLC

\section{Introduction}

Heme, iron protoporphyrin IX, is a well known essential molecule for living organisms, particularly for humans [1]. In humans, heme plays an important role in diverse processes, such as erythroid cell biogenesis, pancreatic development, circadian rhythm, and neurogenesis [1,2]. It serves as a prosthetic group in various enzymes and proteins involved in oxygen utilization and metabolism, such as hemoglobin, myoglobin, cytochromes, peroxidases and catalases [1,3]. Furthermore, heme is involved in the regulation of transcription, translation and micro-RNA biogenesis [1]. Given the important role of heme in various processes, it is conceivable that alterations in heme metabolism are associated with various diseases and disorders in humans. A decrease in heme levels is associated with porphyrias, anemia, and neurological disorders such as Alzheimer's disease [1]. Conversely, high consumption of heme in red meat is associated with increased risk of various diseases; such as Type2 diabetes, coronary heart disease, and cancers including colorectal cancer, pancreatic cancer and lung cancer [4]. An independent study which used the whole genome expression analysis has established a link between high heme intake and lung carcinogenesis. They found that $\sim 28 \%$ of the annotated genes were involved in heme transport, absorption, binding, biosynthesis and heme signaling pathway [5].

Lung cancer is the leading cause of mortality among all cancers, in the US and worldwide [6]. It accounts for about 1.2 million deaths every year [6]. Despite the progress in lung cancer treatment and diagnosis, the prognosis is still very poor, with a 5-year survival rate of around 15\% [7]. Therefore, understanding the molecular link between heme function and lung cancer progression may identify novel strategies for cancer diagnosis and treatment. Work in our lab has revealed the association between heme function and lung cancer progression by using a matched pair of lung cell lines: non-malignant normal (HBEC30KT) cells and non-small-cell lung cancer cells (HCC4017). The proteomic analysis in our lab has shown that the levels of a number of hemoproteins are increased in cancer vs. normal cells (Table 1) [8] We found that the levels of proteins involved in both heme biosynthesis (ALAS1) and heme uptake (HCP1 and HRG-1), and the levels of oxygen-utilizing hemoproteins, such as cytochromes and cytoglobins, are upregulated in NSCLC cells compared to normal cells [10]. We also found that the rates of oxygen consumption are elevated in cancer cells compared to normal cells. Additionally, we examined the effect of glucose and glutamine, the two major nutrients for cancer cells, on oxygen consumption. Overall, our data reveal a key role of heme in the progression of lung cancer. This may lead to new strategies in the diagnosis and treatment of lung cancer.

\section{Materials and Methods}

\section{Lung cell lines, cell count and reagents}

HBEC30KT and HCC4017 cell lines representing normal nonmalignant and NSCLC cells were provided by Dr. John Minna's lab (UTSW) as a gift $[9,22]$. They were developed from the same patient and were maintained in ACL4 supplemented with 2\% FBS under 5\% $\mathrm{CO}_{2}$ at $37^{\circ} \mathrm{C}$ [9]. All other NSCLC cell lines including H1395, H1299, Calu-3, A549, H2009 and H460 were purchased from ATCC, and were maintained in RPMI1640 with 5\% FBS. All tissue culture medium,

*Corresponding author: Zhang L, Department of Molecular and Cell Biology, Center for Systems Biology, University of Texas at Dallas, Mail Stop RL11, 800 W. Campbell Road, Richardson, TX 75080, USA, Tel: 972-883-5757; E-mail: li.zhang@utdallas.edu

Received August 22, 2015; Accepted September 09, 2015; Published September 11,2015

Citation: Hooda J, Alam MM, Zhang L (2015) Evaluating the Association of Heme and Heme Metabolites with Lung Cancer Bioenergetics and Progression. Metabolomics 5: 150. doi:10.4172/2153-0769.1000150

Copyright: $\odot 2015$ Hooda J, et al. This is an open-access article distributed unde the terms of the Creative Commons Attribution License, which permits unrestricted use, distribution, and reproduction in any medium, provided the original author and source are credited. 


\begin{tabular}{|c|c|c|c|c|}
\hline \multicolumn{4}{|c|}{ A List of Mitochondrial Proteins Upregulated in NSCLC Cells } \\
\hline Protein ID & Gene Symbol & \multicolumn{1}{c|}{ Description } & Fold \\
\hline NP_001203 & C1QBP & complement 1Q subcomponent-binding proteins & 1.5 \\
\hline NP_001907 & CYC1 & cytochrome c1, heme protein & $3.27 \mathrm{E}-02$ \\
\hline NP_002147 & HSPD1 & 60 kDa heat shock protein & 3.6 \\
\hline NP_573566 & LRPPRC & leucine-rich PPR motif-containing protein & 3.3 \\
\hline NP_006285 & UQCRB & cytochrome b-c1 complex subunit 7 & 3.9 \\
\hline
\end{tabular}

Table 1: A list of mitochondrial proteins upregulates in NSCLC cells [8].

including those lacking glucose or glutamine, were purchased from Invitrogen Life Technologies. Heme - deficient medium was prepared as described before [2]. For measuring the effect of reagents on lung cell proliferation, cells were seeded in 48 -well plate at a density of $10^{3}$ cells/ well. After culturing for $24 \mathrm{~h}$, cells were treated with $0.5 \mathrm{mM}$ succinyl acetone. Every $24 \mathrm{~h}$ post treatment, the number of live cells was counted by using trypan blue staining and a hemocytometer.

\section{Measurement of oxygen consumption rates and heme synthesis}

NSCLC cells ( $\sim 70 \%$ confluence) were maintained in respective medium or in medium lacking glucose or glutamine for $24 \mathrm{~h}$. Then, oxygen consumption was measured, as described previously [10]. Briefly, cells with about $70 \%$ confluency were trypsinized and resuspended in fresh, air-saturated medium. For each measurement, $10^{6}$ cells (in $350 \mu \mathrm{l}$ ) were introduced in the chamber of an Oxygraph system (Hansatech Instruments), with a Clark-type electrode placed at the bottom of the respiratory chamber. During measurements, the chamber was thermostated at $37^{\circ} \mathrm{C}$ by a circulating water bath. An electromagnetic stirrer bar was used to mix the contents of the chamber. Each measurement was replicated at least three times. Heme synthesis rate was measured as described previously $[2,11]$. Briefly, cells were treated with $0.5 \mathrm{mM}$ succinyl acetone for $48 \mathrm{~h}$, and were incubated with $0.5 \mu \mathrm{Ci} / \mathrm{ml}$ of $\left[4-{ }^{14} \mathrm{C}\right] 5$-aminolevulinic acid (PerkinElmer Life and analytical Sciences) for $15 \mathrm{~h}$. Heme was extracted from these cells with acetone-hydrochloric acid and diethyl ether, and the amount of radiolabeled heme was measured using scintillation counter. Standard deviations were calculated, and $\mathrm{p}$ values were calculated by using Welch 2-sample t-test.

\section{Preparation of protein extracts and western blotting}

NSCLC cells were treated, collected, and lysed by using the RIPA buffer (Cell Signaling Technology) containing the protease inhibitor cocktail. Human tumor xenografts were collected, and lysates from human tumor xenografts were prepared as described [12]. Protein concentrations were determined by using the BCA assay kit (Thermo Scientific). Fifty micrograms of protein from each treatment condition were electrophoresed on $9 \%$ SDS-Polyacrylamide gels, and then transferred onto the Immuno-Blot PVDF Membrane (Bio-Rad). The membranes were probed with polyclonal antibodies, followed by detection with a chemiluminescence Western blotting kit (Roche Diagnostics). The signals were detected by using a Carestream image station 4000 MM Pro, and quantitation was performed by using the Carestream molecular imaging software version 5.0.5.30 (Carestream Health, Inc.). Polyclonal anti-ALAS1 (sc-50531), anti-cytoglobin (sc66855), and anti-HCP1 (sc-134997) were purchased from Santa Cruz Biotechnology. Monoclonal anti- $\beta$-actin (4967) antibody was purchased from Cell Signaling Technology.

\section{Colony formation assay}

Colony formation assay was performed by counting HCC4017 cells and seeding in 6-well plates at a density of 1000 cells per well. Cells were treated without or with $0.5 \mathrm{mM}$ succinyl acetone, succinyl acetone + heme $(10 \mu \mathrm{M}), 10 \mu \mathrm{M}$ Tin protoporphyrin IX (SnPP) or 10 $\mu \mathrm{M}$ carbonyl cyanide $\mathrm{m}$-chlorophenyl hydrazone (CCCP). Medium was changed every three to four days. After a period of 10-12 days, cells were fixed in $70 \%$ ethanol and stained with $0.5 \%$ crystal violet. The images were acquired by using the HP Scanjet 8270 [10].

\section{Results}

NSCLC cells have intensified levels of heme biosynthesis and heme biosynthetic enzymes

We measured the levels of heme synthesis rate in normal and NSCLC cells. Interestingly, the levels of heme synthesis were increased in NSCLC cells compared to normal cells (Figure 1A). In the presence of succinyl acetone (SA), a potent inhibitor of heme synthesis which targets the second enzyme in the heme synthesis pathway ALAD ( $\delta$ - aminolevulinic acid dehydratase), the levels of heme synthesis decreased in both normal and cancer cells as expected (Figure 1A) [10]. Reasoning that the increased levels of heme synthesis could be associated with the increased levels of heme biosynthetic enzymes, we measured the levels of ALAS1 (5-aminolevulinic acid synthase 1), a rate limiting enzyme in heme synthesis pathway in non-erythroid cells including lung cells. ALAS1 is the housekeeping gene, and the expression levels are controlled by the levels of heme [13]. We found that the levels of ALAS1 were intensified in cancer cell compared to normal cells (Figure 1B), and as expected, the levels of ALAS1 enzyme were further enhanced in the presence of SA in both normal and cancer cells (Figure 1B). Further, we measured the levels of ALAS1 enzyme in various human tumor xenografts. Consistent with the cell line data, the levels of ALAS1 enzyme were found to be intensified in xenograft samples (Figure 1C). These results have, for the first time, identified increased levels of heme biosynthesis and heme biosynthetic enzymes in lung cancer cells with direct comparison to lung normal cells.

\section{NSCLC cells have increased levels of heme uptake proteins and oxygen-utilizing hemoproteins}

High demand for heme in cancer cells can be met either by increased levels of heme synthesis as shown in Figure 1, or by increased levels of heme uptake. Therefore, we measured the levels of two known heme uptake proteins, proton-coupled folate transporter/heme carrier protein 1 (PCFT/HCP1) and heme-resposive gene 1 (HRG-1). HCP1 is a low affinity heme transporter. It is highly expressed in the duodenal mucosa, and plays a key role in the intestinal absorption of heme and folate from diet $[4,14]$. We found that the levels of HCP1 protein were intensified in NSCLC cells (HCC4017) (Figure 2A). Similarly, the levels of HRG-1 protein were intensified in NSCLC cell compared to 


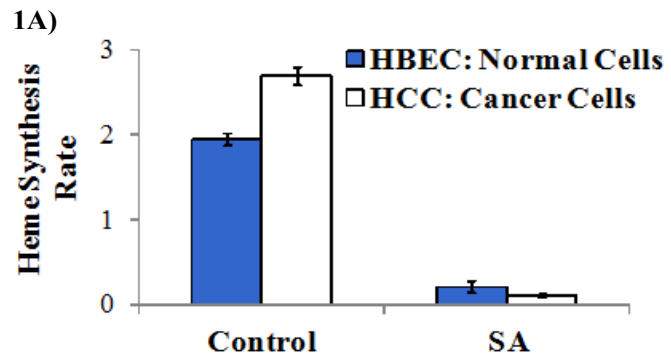

1B)
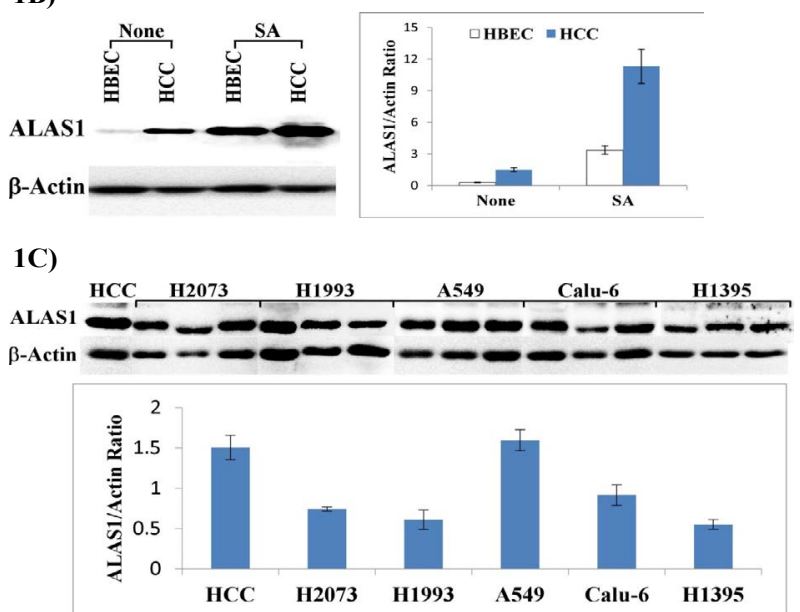

Figure 1: Rate of heme synthesis and the levels of heme biosynthetic enzymes are elevated in NSCLC cells. (A) Rate of heme synthesis in cancer (HCC4017) vs. normal (HBEC30KT) cells, in absence or presence of $0.5 \mathrm{mM}$ succinyl acetone (SA). (B) Protein levels of ALAS1 in HBEC30KT and HCC4017 cell lines in absence or presence of $0.5 \mathrm{mM}$ succinyl acetone (SA). (C). Increased levels of ALAS1 enzyme in HCC4017 (HCC) cell line and various human tumor xenograft samples [10].

normal cells (Data not shown) [10]. Interestingly, inhibiting the heme synthesis in cancer cells with SA further increased the levels of HCP1 (Figure 2A). HCP1 levels were similarly intensified in various human tumor xenograft samples (Figure $2 \mathrm{~B}$ ).

Heme serves as a prosthetic group in various hemoproteins including cytochromes and cytoglobins; increased heme synthesis and uptake can provide additional heme for the production of hemoproteins. Cytoglobin is a hemoprotein facilitating oxygen transport. We found that the levels of cytoglobin were highly intensified in NSCLC cells, while it was not detected in normal cells (Figure 2C). Further, inhibiting heme synthesis using SA decreased the levels of cytoglobin, and an addition of heme in the medium reversed the levels of cytoglobin (Figure 2C). Likewise, the levels of cytoglobin were highly intensified in the tumor xenograft samples (Figure 2D). Similarly, the levels of other hemoproteins including cytochrome c, CYP1B1 and Cox-2, were enhanced in NSCLC cells compared to normal cells (Data not shown) [10].

\section{Oxygen consumption is intensified in NSCLC cells, and it is suppressed in heme-deficient cells}

Heme plays a vital role in oxygen utilization and sensing in virtually all organisms [2,15]. We showed that the levels of oxygen-utilizing hemoproteins are intensified in NSCLC cells. Therefore, we measured the oxygen consumption rate of NSCLC cells, and the effect of heme depletion on the rate of oxygen consumption. We found that cancer cells (HCC4017) have elevated levels of oxygen consumption compared to normal cells (HBECK30KT) (Figure 3). Further, inhibiting heme synthesis using SA or depletion of heme in the medium significantly decreased the oxygen consumption in cancer cells. Interestingly, heme depletion affects the oxygen consumption in NSCLC cells more severely than in HBEC30KT cells (Figure 3). We also measured the oxygen consumption rates in the presence of CCCP, which is a mitochondrial uncoupler. As expected, the presence of CCCP decreases the levels of oxygen consumption in both normal and cancer cells.

\section{Reduction of intracellular heme levels inhibits NSCLC cell proliferation and colony formation}

We further evaluated the effect of heme synthesis and mitochondrial function on cancer cell proliferation and colony formation. Figure $4 \mathrm{~A}$ shows that inhibition of heme synthesis reduces the proliferation of HCC4017 cells. The effect of heme inhibition was more severe on NSCLC cells proliferation than HBEC30KT cells (Figure 4A). Likewise, heme inhibition stopped the colony formation of cancer cells, and

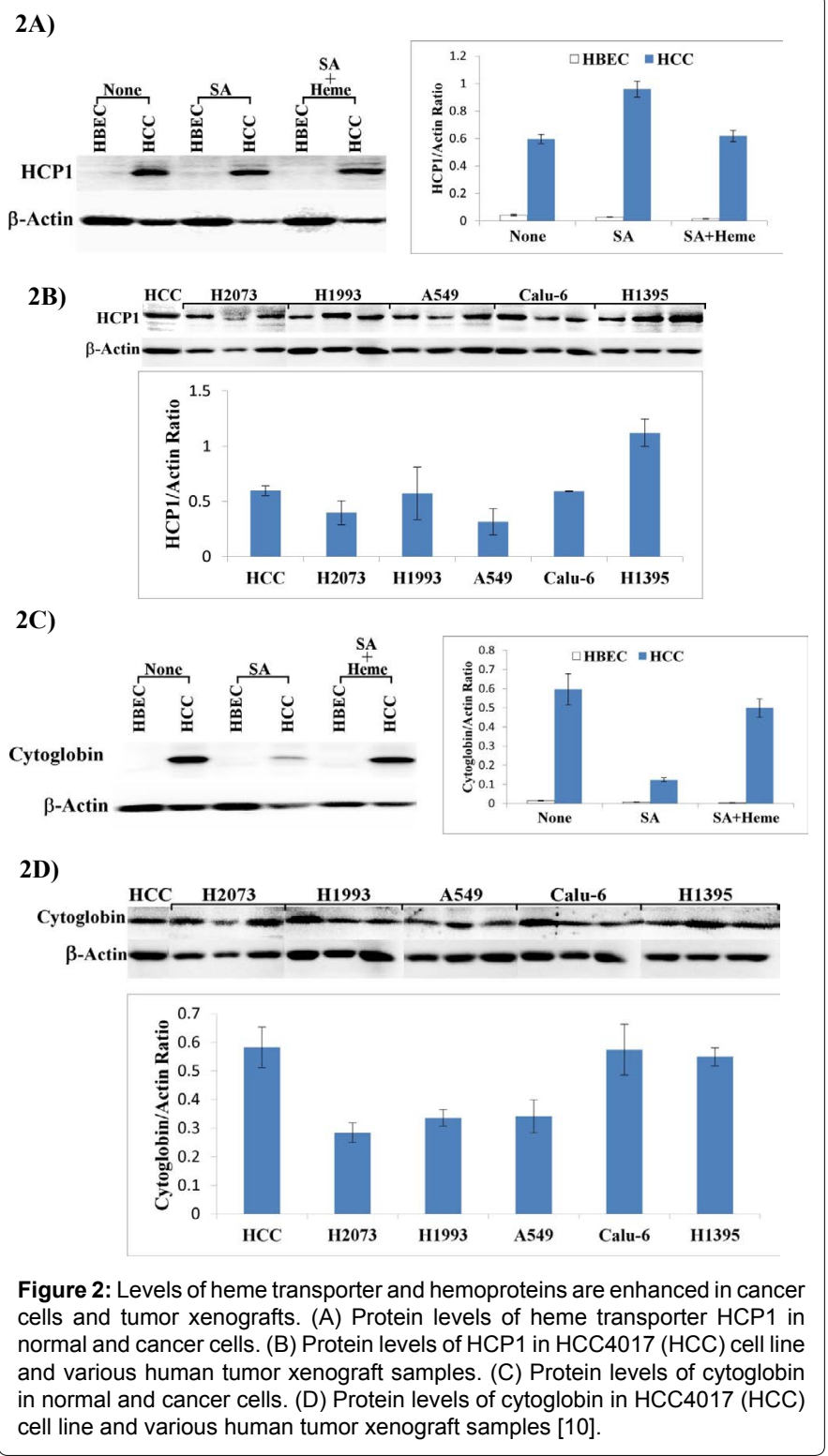




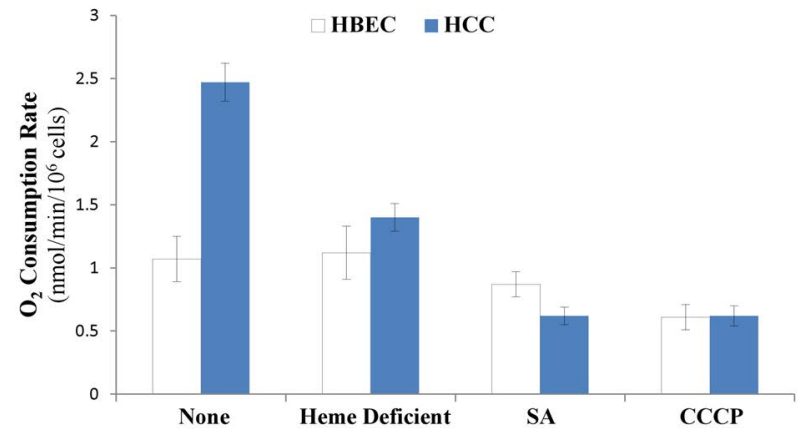

Figure 3: Increased rates of oxygen consumption in cancer cells (HCC) compared to normal (HBEC) cells. Heme depletion (heme deficient) in medium and/or inhibition of heme synthesis (SA) reduces the rates of oxygen consumption in cancer cells [10].

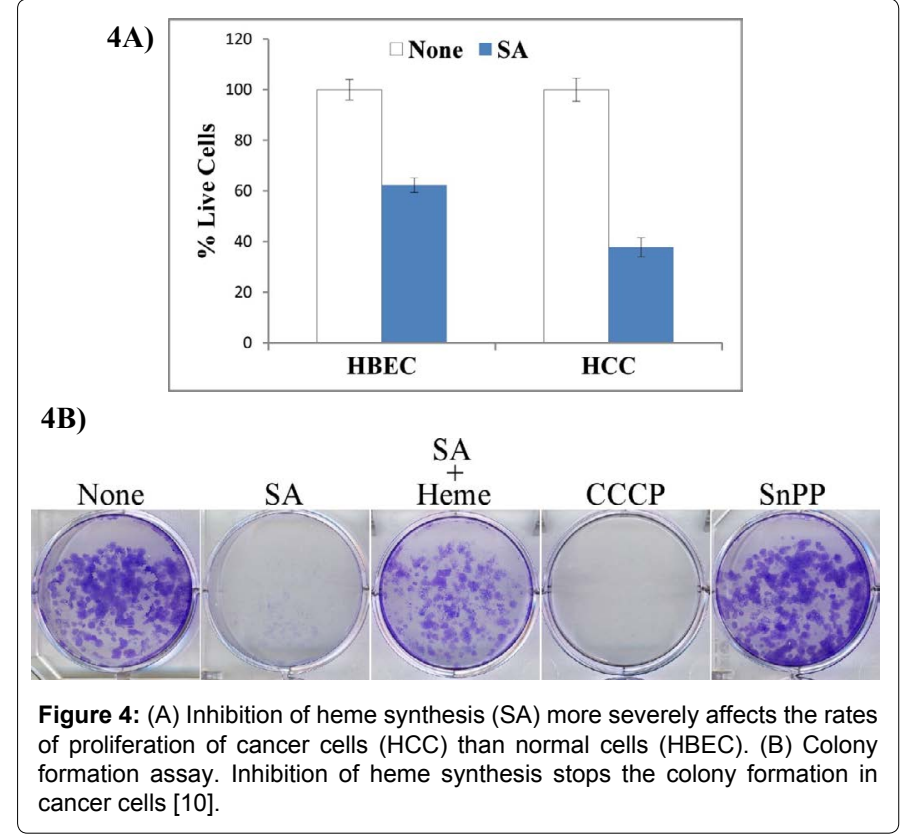

addition of heme in the medium recovered colony formation (Figure 4B). As expected, CCCP also stopped colony formation by cancer cells. In contrast, inhibition of heme degradation by SnPP did not have an effect on colony formation.

\section{Various NSCLC cell lines exhibit intensified levels of oxygen consumption and heme synthesis}

Figure 5A shows that the levels of oxygen consumption are elevated in all NSCLC cells, compared to nontumorigenic HBEC30KT cells. Similarly, we found that the levels of heme synthesis were intensified in the NSCLC cells (Figure 5B). The increase in heme biosynthesis and oxygen consumption promotes the progression of NSCLC cells.

\section{Glucose and Glutamine deprivation strongly impacts the rate of oxygen consumption in NSCLC cells}

We measured the rate of oxygen consumption under glucose and glutamine starvation in four NSCLC cell lines including HCC4017, A549, H1299 and H1395. Cells were subjected to glucose and glutamine starvation by maintaining them in glucose- or glutamine-free medium for $24 \mathrm{~h}$. In all the four NSCLC cell lines, glucose and glutamine deprivation had opposite effects on oxygen consumption rates. In glucose-deprived medium, oxygen consumption is significantly increased, while in glutamine-deprived medium, it is substantially decreased (Figure 6).

\section{Discussion}

Cell proliferation and metabolism are intimately connected. The potential difference between the metabolism of normal and cancer cells has recently gained intense interest in the discovery of new targets for cancer treatment [16]. Altered metabolism is indeed a hallmark of cancer, and a tremendous effort has been put to understand cancer cell metabolism. Despite the extensive research in cancer metabolism, the role of heme metabolism in cancer progression is poorly understood. Here we have shown that heme metabolism is altered in NSCLC cells, and heme plays a critical role in the progression of lung cancer. To meet the high demand for heme, cancer cells and human tumor xenografts show intensified levels of heme biosynthetic enzyme (ALAS1) and heme uptake proteins (HCP1 and HRG-1), as shown in Figures $1 \mathrm{~B}$ and $1 \mathrm{C}$ and Figures $2 \mathrm{~A}$ and $2 \mathrm{~B}$ respectively [10]. This leads to increased levels of heme synthesis in cancer cells compared to normal cells (Figure 1A). Our study has focused on an individual metabolite, heme. Recent metabolomic techniques have provided a platform to identify and compare the metabolites on a global scale, instead focusing on a

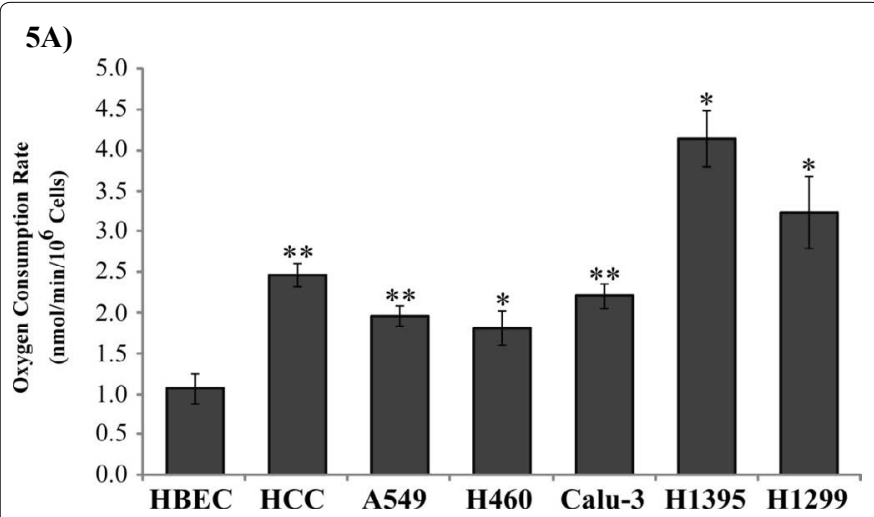

5B)

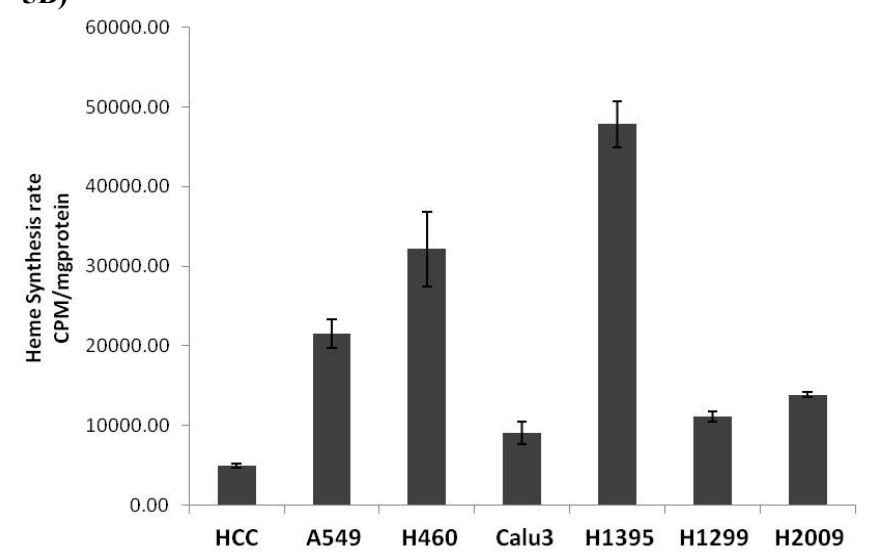

Figure 5: The rates of oxygen consumption and heme synthesis are increased in NSCLC cells. Differential levels of rates of oxygen consumption (A), and heme synthesis (B) in various NSCLC cell lines. For statistical analysis, the levels in cancer cells were compared to the levels in normal cells (HBEC), by using Welch 2 -sample t-test. * $p$ value $0.05 ;{ }^{* *} p$ value 0.005 . 
single metabolite [17]. These techniques could be applied to compare the levels of heme biosynthetic intermediumtes to provide further details between the heme metabolism of normal and cancer cells. Measuring the levels of heme biosynthetic intermediates is challenging, because they are rapidly delivered to the next enzyme in the pathway due to the toxicity of these intermediates to the cell [18]. We also attempted to measure the levels of heme biosynthetic intermediates using metabolomic techniques; however, the results were inconclusive. Heme plays diverse roles in cells. It is a prosthetic group in oxygenutilizing hemoproteins including cytoglobins and cytochrome $c$, and it acts as a regulator, modulating transcription, translation and microRNA biogenesis [1]. In cancer cells and tumor xenografts, an increase in heme synthesis and uptake occurs concomitantly with increased levels of hemoproteins such as cytoglobin (Figure 2C), cytochrome c, Cox-2 and CYP1B1. Cytoglobin is able to bind oxygen, and it is known to regulate oxygen levels in cancer cells during hypoxia [19]. Cytoglobin is a stress-related gene, and its expression level is upregulated during oxidative stress in tumors [19]. Our data has shown that the level of cytoglobin is increased many folds in NSCLC cells and tumor xenografts (Figure $2 \mathrm{C}$ and 2D). Furthermore, our data indicate that the levels of cytoglobin are controlled directly or indirectly by the levels of heme in the cancer cells (Figure 2) [10].

Recent research suggests that mitochondrial respiration is a major source of energy supply in various human cancer cells [20]. Furthermore, it has been shown that cancer cells can maintain substantial levels of oxidative phosphorylation even under hypoxia [21]. Given that the levels of heme and hemoproteins are highly elevated in NSCLC cells and that $\mathrm{K}_{\mathrm{m}}$ for $\mathrm{O}_{2}$ of various heme biosynthetic enzymes and mitochondrial respiratory hemoproteins is very low (micromolar range). It is likely that even under hypoxia, cancer cells generates high levels of heme and hemoproteins, maintaining high levels of oxidative phosphorylation, thereby sustaining cancer cell proliferation and progression. Furthermore, our results show that glutamine is the main fuel for generating cellular energy supply in cancer cells, as shown previously. Our results show that in absence of glucose, glutamine supports intensified oxygen consumption in cancer cells. In the absence of glutamine, even in the presence of glucose, oxygen consumption is substantially reduced (Figure 6)

\section{Conclusions}

Our study has revealed key bioenergetics alterations in cancer cells compared to normal cells. We have shown that the rate of heme synthesis, levels of heme biosynthetic enzyme (ALAS1) and heme uptake proteins are elevated in cancer cells (Figure 7). Increased availability of heme in NSCLC cells enhances the levels of key hemoproteins in mitochondria, which are involved in oxidative phosphorylation, and the levels of oxygen-utilizing hemoproteins in other cellular compartments. Such elevated levels of heme and hemoproteins enable cancer cells to increase the mitochondrial respiration, which provides ample energy to fuel the progression of NSCLC cells (Figure 7). Interestingly, limiting the cancer cells' access to heme decreases their proliferation, colony formation and oxygen consumption rate. Our data strongly suggests that heme is a key stimulator in cancer cell progression, and this can provide a new strategy in cancer diagnosis and treatment. Furthermore, glucose and glutamine are two key nutrients for cancer cells, and glucose starvation in the presence of glutamine elevates the levels of oxygen consumption, whereas glutamine starvation in the presence of glucose decreases the levels of oxygen consumption in NSCLC cells [22,23].

\section{References}
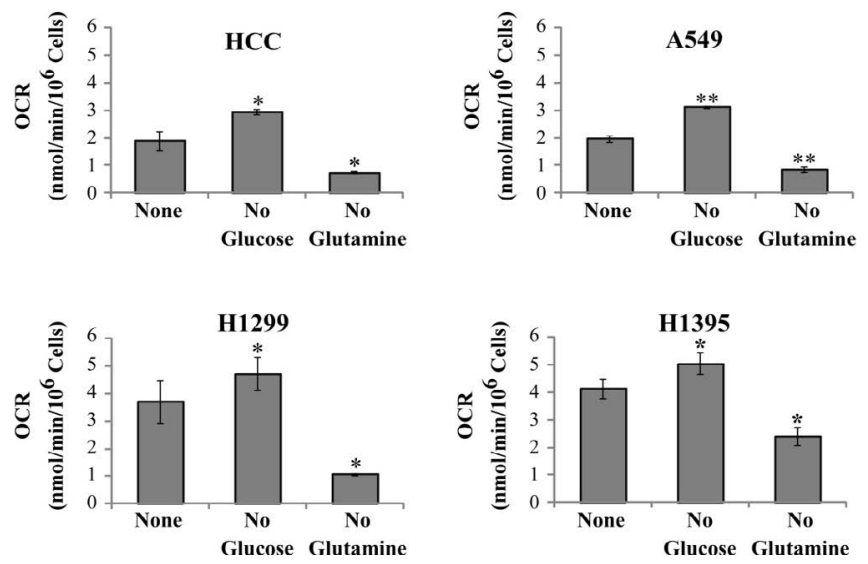

Figure 6: The effect of glucose and glutamine deprivation on the rate of oxygen consumption in NSCLC cells. The four NSCLC cell lines were cultured in regular medium or the medium lacking glucose or glutamine for $24 \mathrm{~h}$, as indicated. The rates of oxygen consumption were measured. The data shown were averages of at least three independent measurements. For statistical analysis, the values were compared to those in normal culture medium (none), by using Welch 2-sample t-test. * $p$ value $<0.05 ;{ }^{* *} p$ value $<0.005$.

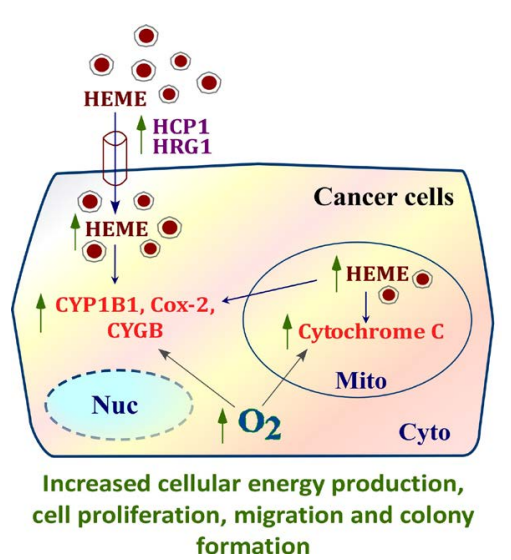

Figure 7: Cartoon illustrating key bioenrgetic changes in NSCLC cells. Cancer cells exhibit enhanced expression levels of the rate-limiting heme biosynthetic enzyme, 5-aminolevulinic acid synthase (ALAS), and the heme uptake proteins HCP1 and HRG-1. The increased heme availability in cancer cells leads to increased levels of various oxygen-utilizing hemoproteins, such as cytochrome c, cytoglobin, Cox-2 and cytochrome P450. The increase in these hemoproteins ultimately leads to intensified oxygen consumption, which provides ample energy to fuel the progression of NSCLC cells. [4,10].

1. Zhang L (2011) HEME BIOLOGY: The Secret Life of Heme in Regulating Diverse Biological Processes. Singapore: World Scientific Publishing Company.

2. Zhu Y, Hon T, Ye W, Zhang L (2002) Heme deficiency interferes with the Rasmitogen-activated protein kinase signaling pathway and expression of a subse of neuronal genes. Cell Growth Differ 13: 431-439.

3. Ya X, Balamurugan P, Arvey A, Leslie C, Zhang L (2010) Heme controls the regulation of protein tyrosine kinases Jak2 and Src. Biochem Biophys Res Commun 403: 30-35.

4. Hooda J, Shah A, Zhang L (2014) Heme, an essential nutrient from dietary proteins, critically impacts diverse physiological and pathological processes Nutrients 6: 1080-1102.

5. Lam TK, Rotunno M, Ryan BM, Pesatori AC, Bertazzi PA, et al. (2013) Hemerelated gene expression signatures of meat intakes in lung cancer tissues. Mol Carcinog 53: 548-556.

6. Lv XJ, Zhao LJ, Hao YQ, Su ZZ, Li JY, et al. (2015) Schisandrin B inhibits the 
Citation: Hooda J, Alam MM, Zhang L (2015) Evaluating the Association of Heme and Heme Metabolites with Lung Cancer Bioenergetics and Progression. Metabolomics 5: 150. doi:10.4172/2153-0769.1000150

proliferation of human lung adenocarcinoma A549 cells by inducing cycle arrest and apoptosis. Int J Clin Exp Med 8: 6926-6936.

7. Kim Y, Kim DH (2015) CpG island hypermethylation as a biomarker for the early detection of lung cancer. Methods Mol Biol 1238: 141-171.

8. Cadinu D, Hooda J, Alam MM, Balamurugan P, Henke RM, et al. (2014) Comparative proteomic analysis reveals characteristic molecular changes accompanying the transformation of nonmalignant to cancer lung cells. EuPA Open Proteomics 3: 1-12.

9. Whitehurst AW, Bodemann BO, Cardenas J, Ferguson D, Girard L, et al (2007) Synthetic lethal screen identification of chemosensitizer loci in cancer cells. Nature 446: 815-819.

10. Hooda J, Cadinu D, Alam MM, Shah A, Cao TM, et al. (2013) Enhanced heme function and mitochondrial respiration promote the progression of lung cancer cells. PLoS One 8: e63402.

11. Hooda J, Alam MM, Zhang L (2015) Measurement of Heme Synthesis Levels in Mammalian Cells. J of Vis Exp 101: e51579.

12. Sullivan LA, Caron JG, Roland CL, Toombs JE, Nyquist-Andersen M, et al (2010) r84, a novel therapeutic antibody against mouse and human VEGF with potent anti-tumor activity and limited toxicity induction. PLoS One 5: e12031.

13. Anderson KE, Sassa S, Bishop DF, Desnick RJ (2001) Disorders of heme biosynthesis: X-linked sideroblastic anemia and the porphyrias. The McGrawHill Companies, New York.

14. Shayeghi M, Latunde-Dada GO, Oakhill JS, Laftah AH, Takeuchi K, et al. (2005) Identification of an intestinal heme transporter. Cell 122: 789-801.
15. Zhang L, Hach A, Wang C (1998) Molecular mechanism governing heme signaling in yeast: A higher-order complex mediumtes heme regulation of the transcriptional activator HAP1. Mol Cell Biol 18: 3819-3828.

16. Comerford SA, Huang Z, Du X, Wang Y, Cai L, et al. (2014) Acetate dependence of tumors. Cell 159: 1591-1602.

17. Sun L, Li J, Zhou K, Zhang M, Yang J, et al. (2013) Metabolomic analysis reveals metabolic disturbance in the cortex and hippocampus of subchronic MK-801 treated rats. PLoS One 8: e60598.

18. Hamza I, Dailey HA (2012) One ring to rule them all: Trafficking of heme and heme synthesis intermediumtes in the metazoans. Biochim Biophys Acta 1823 1617-1632.

19. Chakraborty S, John R, Nag A (2014) Cytoglobin in tumor hypoxia: Nove insights into cancer suppression. Tumour Biol 35: 6207-6219.

20. Bonuccelli G, Tsirigos A, Whitaker-Menezes D, Pavlides S, Pestell RG, et al. (2010) Ketones and lactate "fuel" tumor growth and metastasis: Evidence that epithelial cancer cells use oxidative mitochondrial metabolism. Cell Cycle 9 : 3506-3514.

21. Frezza C, Zheng L, Tennant DA, Papkovsky DB, Hedley BA, et al. (2011) Metabolic profiling of hypoxic cells revealed a catabolic signature required for cell survival. PLoS One 6: e24411.

22. Ramirez RD, Sheridan S, Girard L, Sato M, Kim Y, et al. (2004) Immortalization of human bronchial epithelial cells in the absence of viral oncoproteins. Cance Res 64: 9027-9034.

23. Whitaker-Menezes D, Martinez-Outschoorn UE, Flomenberg N, Birbe RC Witkiewicz AK, et al. (2011) Hyperactivation of oxidative mitochondrial metabolism in epithelial cancer cells in situ: Visualizing the therapeutic effects of metformin in tumor tissue. Cell Cycle 10: 4047-4064. 